\title{
Userperspektive auf Brand Content: Der Uses-and-Gratifications-Ansatz
}

Im vorangegangenen Kapitel über «Marke» wurde unter anderem auf die Markenkommunikation eingegangen. Die klassische Markenkommunikation folgt einer Unternehmensperspektive, anhand welcher funktionale oder emotionale Nutzen vermittelt werden und ein entsprechendes Markenimage aufgebaut werden soll. Durch die Informationsflut und ein selbstbestimmtes Userverhalten im digitalen Raum muss sich Markenkommunikation jedoch zunehmend an den Usern ausrichten und diesen einen Mehrwert bieten. Konzepte wie Content Marketing oder Brand Content behandeln diese Thematik. Allerdings, das wurde im vorherigen Kapitel ebenfalls dargelegt, fehlt sowohl eine präzise Beschreibung dieses Mehrwertes als auch eine systematische Konsolidierung mit der Unternehmensperspektive.

Dieses Kapitel fokussiert sich deshalb auf die Userperspektive, um diese beiden Lücken im Konzept Brand Content zu klären. Dazu eignet sich der Usesand-Gratifications-Ansatz (U\&G-Ansatz; H. Schramm \& Knoll, 2013; Tropp, 2019). Dieser befasst sich mit den Gratifikationen der Mediennutzung, d. h. mit den individuellen Bedürfnissen oder Motiven der Mediennutzung und der Befriedigung dieser Bedürfnisse oder Motive (W. Schweiger, 2007, S. 60). Sind diese Motive bekannt, können auch Marken ihre Kommunikation daran ausrichten und auf erhöhte Aufmerksamkeit der User ihren Botschaften gegenüber hoffen (Tropp, 2019).

Der U\&G-Ansatz hat im Rahmen dieser Arbeit einen zweiten Zweck. Es wird im weiteren Verlauf dieses Kapitels dargelegt, dass Menschen gegenüber Medien Erwartungshaltungen bilden auf Basis der primären, zu erwartenden Gratifikationen (LaRose \& Eastin, 2004). Diese Gratifikationserwartungen bilden einen medienspezifischen Kontext, der die Wirkung von Brand Content beeinflussen kann. Bevor im weiteren Verlauf der Arbeit postuliert werden wird, wie 
ein solcher Kontext wirkt, werden in diesem Kapitel die mit sozialen Medien verbundenen Gratifikationserwartungen thematisiert.

\subsection{Einordnung und Hintergrund}

Die frühe Kommunikationsforschung bis ca. 1950 basierte auf der Annahme starker und einflussreicher Medien und untersuchte deren Einwirken auf Meinungen, Einstellungen und Verhalten der Menschen (Katz, 1959; Schenk, 2007, S. 7). Dieser Annahme nach verarbeiten Menschen Medienbotschaften in passiver und unkritischer Manier. Medien können dadurch Menschen, deren Meinungen und deren Verhalten direkt beeinflussen (Quan-Haase \& Young, 2014, S. 270). Die Leitfrage dieser klassischen, wirkungsorientierten Forschung war gemäss Katz (1959, S. 2): «What do the media do to the people?» Als sich mit zunehmender Forschung herausstellte, dass die Medienwirkungen weniger stark waren als ursprünglich angenommen, rückten neue Perspektiven der Kommunikationsforschung in den Fokus - unter anderem der Uses-and-Gratifications-Ansatz (Katz, 1959). Dem U\&G-Ansatz liegt ein Modell der «limitierten Effekte» (S. 155) zugrunde (Palmgreen \& Rayburn II, 1979). Im Zentrum steht die Leitfrage: «What do people do with the media?» (Katz, 1959, S. 2) Angesichts der neuen Leitfrage ergibt sich ein Perspektivenwechsel: Mit dem U\&G-Ansatz stehen nicht mehr die Medien und deren direkte Einflussnahme auf den Menschen im Vordergrund, sondern der Mensch selbst und seine Zuwendung, bzw. deren Motive, hin zu Medien (Schenk, 2007, S. 681). W. Schweiger (2007, S. 62) weist darauf hin, dass dieser Perspektivenwechsel keinem Paradigmenwechsel gleichkomme. Die Wirkungsforschung bleibe weiterhin bestehen. Während die Wirkungsforschung aber den Kontakt zwischen Medium und Mensch als gegeben annehme, fokussiere sich der U\&G-Ansatz auf das Zustandekommen dieses Kontakts. Dabei wird von einer Abfolge verschiedener Faktoren ausgegangen. Nach Katz et al. (1974) beschäftigt sich der U\&G-Ansatz demnach mit:

(1) the social and psychological origins of (2) needs, which generate (3) expectations of (4) the mass media or other sources, which lead to (5) differential patterns of media exposure (or engagement in other activities), resulting in (6) need gratifications and

(7) other consequences, perhaps mostly unintended ones. (Katz et al., 1974, S. 510)

Haridakis und Whitmore (2006, S. 767) sehen die Anfänge der U\&G-Forschung in den frühen 1940er-Jahren. W. Schramm (1949) etwa untersuchte, wieso sich Leser und Leserinnen Zeitungen zuwenden. Blumler (1979, S. 10) datiert den 
Aufschwung des Ansatzes auf die späten 1950er- und die frühen 1960er-Jahre angekurbelt durch Studien, welche die angenommenen starken Medienwirkungen nicht nachweisen konnten. Für W. Schweiger (2007, S. 60) erreicht der Ansatz seinen Höhepunkt in den 1970er- und 1980er-Jahren vor dem Hintergrund des sich vervielfältigenden Angebots an Kabelfernsehstationen in den USA. Die Frage nach den Mediennutzungsmotiven ist für ihn allerdings so alt «wie die Medien selbst» (W. Schweiger, 2007, S. 60). Weil der Ansatz aber immer dann an Resonanz gewinnt, wenn sich neue Medien verbreiten (W. Schweiger, 2007, S. 60), erlebte er mit dem Aufkommen der Digitalisierung und des Internets eine neue Blüte (Quan-Haase \& Young, 2014, S. 269).

\subsection{Grundannahmen}

Der U\&G-Ansatz basiert auf verschiedenen Grundannahmen, die sich in ähnlicher Ausformulierung bei verschiedenen Autoren finden (Katz et al., 1974, S. 510511; Rubin, 2009, S. 167; Schenk, 2007, S. 684-685). Die folgende Auflistung orientiert sich an W. Schweiger (2007, S. 61-63).

(1) Der ersten Annahme nach handeln Medienrezipierende oder User aktiv und zielgerichtet. Diese Aktivität ist funktional und dient «der Erreichung gewünschter Wirkungen» (W. Schweiger, 2007, S. 61), d. h., die User wählen das Medienangebot gezielt aus, um ihre Bedürfnisse zu befriedigen (Rubin, 2009, S. 167). Die Annahme gilt jedoch nicht uneingeschränkt für sämtliches Mediennutzungsverhalten. Insbesondere seit den 1980er-Jahren wird dem habituellen oder ritualisierten Medienkonsum mehr Beachtung geschenkt (Ruggiero, 2000, S. 8; Schenk, 2007, S. 703-706). Beim habitualisierten oder ritualisierten Konsum wird davon ausgegangen, dass gewisse alltägliche Verhaltensweisen nur bedingt durch Intention, dafür stärker durch Gewohnheit beeinflusst sind (Schenk, 2007, S. 704). Und wo Verhalten durch Gewohnheit ausgelöst wird, deutet das auf ein passives Bild der Nutzer hin (Rosenstein \& Grant, 1997). Motive wie Zeitvertreib, Ablenkung oder Entspannung sind Indikatoren einer passiven Nutzung (Schenk, 2007, S. 705). Präziser ist es deshalb, das Publikum als «differentiell aktiv» (Schenk, 2007, S. 703) zu beschreiben bzw. von einem «Spektrum der Aufmerksamkeit» (Steiner \& Xu, 2020, S. 82) auszugehen. Je nach Motivation des Medienkonsums verfolgen die Zuschauer die Inhalte mehr oder weniger aufmerksam (Steiner \& Xu, 2020). Insbesondere im Umfeld digitaler Medien wird allerdings wieder von einer grösseren Aktivität ausgegangen (Ruggiero, 2000, S. 19). Sundar und Limperos (2013, S. 505) führen dies auf den Charakter der neuen Medien zurück, die mehr Interaktivität zulassen. 
(2) Eine zweite Annahme besagt, dass die Initiative der Mediennutzung beim Menschen liegt (Katz et al., 1974, S. 511). Die Nutzer entscheiden, wann, wie und wo sie welche Medien konsumieren (Schenk, 2007, S. 685). Damit werden Medienwirkungseffekte nicht in Abrede gestellt, es wird aber nicht mehr von direkten Effekten des Inhalts auf Einstellungen oder Verhalten geschlossen (Katz et al., 1974, S. 511).

(3) Es wird, so eine dritte Annahme, davon ausgegangen, dass Nutzerinnen und Nutzer sich ihrer Mediennutzungsmotive bewusst sind. Sie können diese auf Anfrage offen formulieren oder zumindest zu erkennen geben, wenn sie mit entsprechenden Vorschlägen konfrontiert werden (Katz et al., 1974). Tatsächlich basieren U\&G-Studien oftmals auf einem zweistufigen Verfahren, in dem zuerst mit offenen Fragen die Bandbreite möglicher Motive erfasst wird (Itemgenerierung) und nachgelagert - nach Bereinigung der Items - mit einem quantitativen Vorgehen die Motive mit Bezug auf Nutzer- oder Mediengruppen ausgewertet werden (Spinda \& Puckette, 2018). Oft wird auch auf den ersten Schritt verzichtet und auf Items früherer Forschung zurückgegriffen (Leiner, Kobilke, Ruess \& Brosius, 2018). Die Annahme, wonach sich Menschen ihrer Motive bewusst sind, sorgt ebenfalls für kontroverse Diskussionen. Insbesondere die Gründe eines habitualisierten Konsums seien für Menschen schwierig zu erkennen und zu benennen (W. Schweiger, 2007, S. 66).

(4) Menschen haben einer vierten Annahme nach unterschiedlichste Bedürfnisse, wobei einige dieser Bedürfnisse durch Medienkonsum gestillt werden können. Die diversen Möglichkeiten, ein spezifisches Bedürfnis zu stillen, werden als funktionale Alternativen betitelt (Klapper, 1963, S. 521; Lichtenstein \& Rosenfeld, 1983, S. 100). Das Bedürfnis nach Unterhaltung kann beispielsweise durch einen Film, ein Buch, einen Spaziergang oder ein Treffen mit Freunden befriedigt werden (Lichtenstein \& Rosenfeld, 1983, S. 100). Medien konkurrieren dementsprechend sowohl untereinander als auch mit anderen funktionalen Alternativen darum, Bedürfnisse der Menschen zu stillen. Unterschiedliche Medien sind dabei unterschiedlich geeignet, bestimmte dieser Bedürfnisse zu befriedigen (Lichtenstein \& Rosenfeld, 1983, S. 100). Dieser letzte Punkt ist im Rahmen der Arbeit von Bedeutung. Es wird argumentiert, dass soziale Medien, die unterschiedliche primäre Bedürfnisse befriedigen, einen unterschiedlichen Rezeptionskontext schaffen. Der Rezeptionskontext wirkt sich wiederum auf die Wahrnehmung und die Wirkung von Brand Content aus. 


\subsection{Einschränkung und Kritik}

Der U\&G-Ansatz wird in der Literatur nicht unkritisch reflektiert. Kritiker haben dem Ansatz unter anderem fehlenden Theoriestatus vorgeworfen, weil er kein überprüfbares Hypothesengebilde postuliere (W. Schweiger, 2007, S. 65). Befürworter argumentieren hingegen, der Ansatz zeichne sich dadurch aus, dass er aus einer «Anzahl verschiedener Theorien über das Phänomen Uses \& Gratifications» (Blumler, 1979, S. 11) bestehe. Diese Theorien weichen zwar immer wieder voneinander $a b$, würden aber ein gemeinsames Untersuchungsfeld und gemeinsame Konzepte teilen (Blumler, 1979, S. 11-12). In einer ähnlichen Richtung argumentiert auch W. Schweiger (2007, S. 66): Der U\&G-Ansatz sei keine Theorie, könne aber als «Forschungsfeld» oder «Denkansatz» der Theoriebildung dienen. Entsprechend spricht W. Schweiger (2007, S. 65-66) Prozessmodellen der Selektionsentscheidung (z. B. Erwartung-Bewertungs-Ansatz durch Palmgreen \& Rayburn II, 1984, siehe Abschnitt 3.4) den Status einer Theorie zu.

Auch fehlende Erklärungskraft wird dem Ansatz vorgeworfen: Wo die Forschungsresultate aus einer reinen Ansammlung von durch die Medien befriedigten Bedürfnissen bestünden, könne von nichts weiter als von einer Tautologie gesprochen werden: «if movies entertain, then there must be an entertainment need» (Lichtenstein \& Rosenfeld, 1983, S. 100). Für Klapper (1963) reicht es entsprechend nicht, zu sagen, dass Nachrichten konsumiert würden, um besser informiert zu sein. Es gelte auch die Konsequenzen dieser Erkenntnis aufzuzeigen, indem man z. B. darlegt, dass die Rezipienten tatsächlich besser informiert sind. Vor diesem Hintergrund schliesslich muss erklärt werden, auf wen oder was sich diese Konsequenzen beziehen. Wo Konsequenzen bei einer Nutzergruppe A auftreten, seien diese bei einer Gruppe B unter Umständen nicht auszumachen (Klapper, 1963, S. 520-521).

Der Ansatz leidet zudem unter einer terminologischen Vielfalt, die auch heute nicht vollständig geklärt ist. Begriffe wie Motiv, Motivation, Bedürfnis, Gratifikation oder Nutzen werden nach wie vor «üblicherweise synonym benutzt» (W. Schweiger, 2007, S. 75). Dies sei ein Grund, wieso die Unterscheidung von psychischen und sozialen Bedürfnissen einerseits und Mediennutzungsmotiven andererseits nicht gelinge (W. Schweiger, 2007, S. 75). Generell bedarf die kausale Kette von den sozialen oder psychologischen Ursprüngen der Bedürfnisse über Bedürfnisse und Motive hin zum Konsum, wie sie von Katz et al. 1974 systematisiert wurde, weiterer Klärung (Ruggiero, 2000, S. 12; W. Schweiger, 2007, S. 74). Sundar und Limperos (2013, S. 506) werfen den Forschenden diesbezüglich auch vor, sich zu stark auf die Nutzer zu fokussieren und zu ignorieren, 
inwiefern Medien und Technologien Bedürfnisse und Gratifikationen beeinflussen. Laut den Autoren müssen Bedürfnisse nicht a priori vorhanden sein. Sie können sich während des Medienkonsums ausbilden (Sundar \& Limperos, 2013, S. 510). Neue Angebotscharaktere (affordances) durch neue Medien haben so zu neuen Gratifikationen geführt (Sundar \& Limperos, 2013, S. 517).

Im Rahmen dieser Arbeit wird der U\&G-Ansatz verwendet, um über Gratifikationen (a) eine Userperspektive auf Brand Content zu gewinnen und (b) den Rezeptionskontext unterschiedlicher sozialer Medien zu ergründen. Vor diesem Hintergrund erscheint der Ansatz auch unter Berücksichtigung der erwähnten Schwächen gewinnbringend.

\subsection{Gesuchte und erhaltene Gratifikationen}

Im Rahmen dieser Arbeit stehen die Motive der Mediennutzung im Fokus. Auf deren Ursprünge wird nicht weiter eingegangen. Zur begrifflichen Einordnung wird auf die Termini «Gratifications Sought» (GS) und «Gratifications Obtained» (GO), die ab den 1970er-Jahren verwendet wurden (Schenk, 2007, S. 691), zurückgegriffen. Dobos definiert die beiden Begriffe folgendermassen:

Gratifications sought, defined variously as needs, expectations, or motivations for media use, arise from and are shaped by individual characteristics and features of the social environment. Gratifications obtained or need gratifications, on the other hand, refer to the actual fulfillment of these media expectations by available alternatives. (Dobos, 1992, S. 30)

Die Begriffe Gratifications Sought, Bedürfnisse, Motive, Gründe und Motivationen der Medienzuwendung werden in dieser Arbeit synonym verwendet und drücken aus, warum sich Menschen den Medien zuwenden. Anstelle des englischen Begriffs wird auch von gesuchten oder nachgefragten Gratifikationen gesprochen. Wenn zudem der Einfachheit halber schlicht von «Gratifikationen» gesprochen wird, dann sind damit ebenfalls diese Motive der Mediennutzung gemeint. Die tatsächliche Befriedigung dieser Bedürfnisse, also was die Menschen letztlich durch den Medienkonsum erhalten, wird mit Gratifications Obtained bzw. mit erhaltenen Gratifikationen ausgedrückt.

Wenn die Motive oder gesuchten Gratifikationen bekannt sind, wegen derer sich Menschen freiwillig den Medien und deren Inhalten zuwenden, dann können Marken mit ihren kommunizierten Inhalten versuchen, ebenfalls diese Motive zu befriedigen, und damit den Usern einen Mehrwert in der Markenkommunikation 
bieten. Das Resultat, so die Annahme, ist eine erhöhte freiwillige Zuwendung zu den Markeninhalten (Tropp, 2019, S. 380-381). Die erhaltenen Gratifikationen kommen im empirischen Teil der Arbeit zum Zuge, wenn untersucht wird, inwiefern die wahrgenommenen (i. S. v. erhaltenen) Gratifikationen des Brand Contents das Markenimage beeinflussen.

Die Unterteilung in GS und GO diente der Vereinheitlichung der Terminologie, hatte insbesondere aber auch Implikationen auf die weitere Forschungsmethodik des Ansatzes. Durch die Gegenüberstellung von GO und GS konnte eruiert werden, inwiefern ein Medieninhalt die bei ihm gesuchten Gratifikationen tatsächlich befriedigt bzw. inwiefern Anpassungen notwendig sind, um die Bedürfnisse des Publikums besser abzubilden (Schenk, 2007, S. 691). Damit sollte insbesondere auch das Mediennutzungsverhalten besser erklärt und vorhergesagt werden können.

Palmgreen und Rayburn II (Palmgreen \& Rayburn II, 1982; Rayburn II \& Palmgreen, 1984) entwickelten auf der Basis von GS und GO, aber insbesondere unter Verwendung der Theorie des überlegten Handelns nach Fishbein und Ajzen (1975; siehe Abschnitt 2.3.3) den Erwartungs-Bewertungs-Ansatz (siehe Abbildung 3.1). «Belief» bezieht sich auf die individuelle erwartete Wahrscheinlichkeit, einen bestimmten Inhalt (z. B. Sportnachrichten) durch ein Medium zu erhalten. «Evaluation» bildet die persönliche Relevanz dieses Inhalts ab (Rayburn II \& Palmgreen, 1984, S. 538-539). Die Autoren definieren GS als «seeking of a particular outcome mediated by the expectancy of obtaining the outcome, times the evaluation of the outcome» (Palmgreen \& Rayburn II, 1982, S. 565). Die gesuchte Gratifikation (GS) «Sportnachrichten» gegenüber einer spezifischen Online-Plattform ergibt sich entsprechend aus der subjektiven Einschätzung der Wahrscheinlichkeit, mit welcher die Online-Plattform tatsächlich Sportnachrichten anbietet, und der subjektiven Relevanz (Evaluation) eines Angebots an Sportnachrichten durch die Online-Plattform. Damit bekommt GS aus konzeptioneller Sicht hier im Vergleich zur oben genannten Definition einen anderen Bedeutungsinhalt, weil sich GS hier auf ein spezifisches Medium bezieht und die Wahrscheinlichkeit einschliesst, ein bestimmtes Bedürfnis (im Sinne der oben genannten Definition von GS) anhand dieses Mediums befriedigen zu können.

Das Modell ist in dieser Komplexität empirisch schwierig anwendbar, denn die an den Umfragen Teilnehmenden mussten sowohl «Beliefs» und «Evaluations» als auch GS und GO bewerten. Die Unterschiede zwischen den Items für alle vier Konstrukte sind minim (Rayburn II \& Palmgreen, 1984). 


\subsection{Erweiterung durch die sozial-kognitive Theorie}

Eine Weiterentwicklung des U\&G-Ansatzes brachte dessen Kombination mit der sozial-kognitiven Lerntheorie nach Bandura (1986). ${ }^{1}$ Die Forscher um LaRose und Eastin störten sich daran, dass viele internetbezogene Studien nur einen kleinen Teil der Varianz der Mediennutzung über Gratifikationen erklären konnten (LaRose, Mastro \& Eastin, 2001). Die Forschenden setzten sich zum Ziel, einerseits die erklärenden Variablen zu ergänzen und zu präzisieren, andererseits aber auch die Erhebungsmethodik auf Basis der Gegenüberstellung von GS und GO kritisch zu hinterfragen. In diesem Kapitel wird auf diesen zweiten Punkt eingegangen.

Die Autoren machten sich dazu die Parallelen zwischen GS-GO-Modellen und dem enaktiven Lernen in der sozial-kognitiven Lerntheorie (Bandura, 1986) zunutze (LaRose et al., 2001, S. 397). Unter enaktivem Lernen wird Lernen durch Erfahrung verstanden (LaRose et al., 2001, S. 397; Peters, Rickes, Jöckel, Criegern \& van Deursen, 2006, S. 280). Erfahrungen durch Medienkonsum (z. B. die erste Folge der Serie XY gefiel mir sehr gut) formen «outcome expectations» (S. 398) hinsichtlich der Konsequenzen zukünftigen Medienkonsums (auch die weiteren Folgen der Serie XY dürften mir gefallen; LaRose et al., 2001). In Anlehnung an Schenk und Jers (2013) wird der Begriff «outcome expectations» im Weiteren mit Gratifikationserwartungen übersetzt.

Bei Gratifikationserwartungen handelt es sich demnach um momentane Einschätzungen zukünftiger Konsequenzen einer Handlung auf Basis früherer Erfahrungen (LaRose et al., 2001, S. 399). Die Gratifikationserwartungen zukünftigen Verhaltens werden ständig aktualisiert, indem einerseits das eigene Verhalten analysiert und reflektiert wird (enaktives Lernen), aber auch das Verhalten anderer

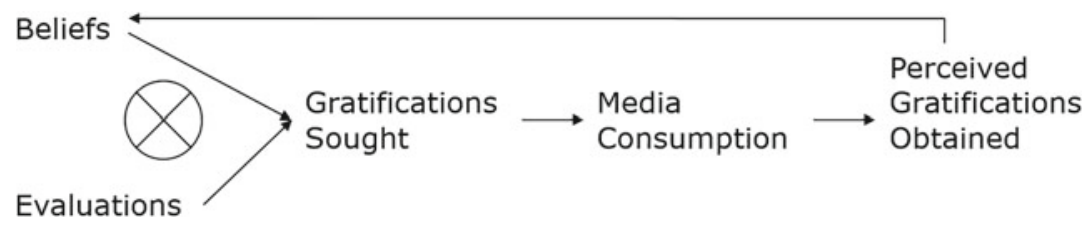

Abbildung 3.1 Expectancy-Value Model of GS and GO. (Nach Rayburn II und Palmgreen (1984, S. 540))

\footnotetext{
${ }^{1}$ Die Abkürzung SC-U\&G für diesen erweiterten Ansatz wurde von Ruehl und Ingenhoff (2017) übernommen.
} 
beobachtet wird (Beobachtungslernen; LaRose \& Eastin, 2004, S. 361; Ruehl \& Ingenhoff, 2015a, S. 290).

Das Handeln des Menschen wird durch Anreize (incentives) wie Hunger, Durst, aber auch Anerkennung, Geld und Status bestimmt. In Bezug auf den Medienkonsum, aber auch bei anderen Handlungen bilden die Gratifikationserwartungen $a b$, inwiefern die Konsequenzen einer Handlung fähig sind, die Anreize zu stillen, bzw. inwiefern eine gewisse Handlung in der Vergangenheit fähig war, die erwarteten Anreize zu stillen (LaRose et al., 2001). Die Anreize im Sinne des SC-U\&G spiegeln damit GS, Motive, Bedürfnisse oder Motivationen in der klassischen U\&G-Terminologie wider.

Die Gratifikationserwartungen schliessen das erwartete Resultat einer Handlung ein und beziehen sich auf ein spezifisches Medium bzw. auf einen spezifischen Medienkonsum. Damit kommt die Definition von Gratifikationserwartungen derjenigen von GS im Sinne von Rayburn II und Palmgreen (1982, S. 565; siehe Abschnitt 3.4) sehr nahe. Während bei Gratifikationen allerdings danach gefragt wird, ob Personen ein Medium für einen bestimmten Zweck nutzen (Rayburn II \& Palmgreen, 1984, S. 545), fragen Gratifikationserwartungen nach der Wahrscheinlichkeit, durch den Medienkonsum ein bestimmtes Ergebnis zu erhalten (LaRose et al., 2001, S. 401).

Die Gratifikationserwartungen der Internetnutzung nach dem SC-U\&G basieren auf sechs Verhaltens-Anreizkategorien (LaRose et al., 2001; LaRose \& Eastin, 2004): Aktivitätsanreize, monetäre Anreize, Neuigkeitsanreize, soziale Anreize, selbstbezogene Anreize und Statusanreize. ${ }^{2}$ Diese sechs Kategorien wurde durch Jers (2012) um die praktischen Anreize und die ideologischen Anreize ergänzt. Jers (2012) untersucht auf Basis der Arbeiten von LaRose und Eastin diejenigen Faktoren, welche «Einfluss darauf nehmen, ob und wie eine Person die verschiedenen Möglichkeiten des Web 2.0 nutzt» (S. 26). Dabei bezieht sie sich einerseits auf die unterschiedlichen Anwendungen des Web 2.0 - namentlich soziale Netzwerke, Wikis, Foren, Blogs, Bilder- und Videoplattformen - und andererseits auf den Interaktionsgrad. Dieser wird in Anlehnung an Shao (2009) in drei Kategorien eingeteilt: Konsum von Inhalten, Partizipation (z. B. in Form von Teilen, Liken oder Kommentieren) und Produktion von Inhalten.

Alle acht Anreizkategorien konnten auch in den Studien von Ruehl und Ingenhoff (2015a, 2017) bestätigt werden. Ruehl und Ingenhoff (2015a) untersuchten anhand semistrukturierter Interviews, wegen welcher Anreize Politiker und Digital Natives über soziale Netzwerke mit Unternehmen interagieren. Qualitative

${ }^{2}$ Die deutschen Übersetzungen wurden von Jers (2012) übernommen. 
Interviewaussagen wurden dabei den Anreizkategorien zugeteilt. In einer weiteren Studie (Ruehl \& Ingenhoff, 2017) standen die Motive im Fokus, anhand welcher User sich auf Facebook Brand Pages engagieren. Für die drei Partizipationslevels nach Shao (2009) - Konsum, Partizipation und Produktion - wurden die relevantesten Anreizkategorien eruiert (Ruehl \& Ingenhoff, 2017).

Es lässt sich zusammenfassen, dass Gratifikationserwartungen primär die Trennung zwischen GS und GO überwinden: «The outcome expectation construct parsimoniously bridges the gulf between gratifications sought and gratifications obtained in uses and gratifications research» (LaRose et al., 2001, S. 399). Das Ziel der Gratifikationserwartungen und der damit verbundenen Messmethodik liegt darin, die Internetnutzung zielgenauer vorauszusagen.

In dieser Arbeit geht es nicht darum, die Internetnutzung zu erklären oder vorherzusagen. Anhand des U\&G-Ansatzes soll unter anderem der Rezeptionskontext digitaler Kanäle definiert werden. Um diesen Rezeptionskontext zu definieren, scheint das Konzept der Gratifikationserwartungen besonders zielführend, weil diese zum Ausdruck bringen, was User von einem Kanal erwarten. Während GS, Motive oder «Gratifikationen» im Rahmen dieser Arbeit Bedürfnisse allgemeiner Art umschreiben (z. B. ich konsumiere Medien, um unterhalten zu werden), verbinden Gratifikationserwartungen diese Gratifikationen unter einer gegebenen Wahrscheinlichkeit mit einem spezifischen Medium oder Medienverhalten (z. B. Facebook hilft mir, mich zu unterhalten). Die Verwendung beider Begriffe im Rahmen dieser Arbeit wird in Abschnitt 3.8 nochmals dargelegt.

\subsection{Allgemeine Gratifikationstypologien}

Im Laufe der Forschung haben sich diverse Forschende mit den Gratifikationen oder Anreizen (SC-U\&G) des Medienkonsums beschäftigt. Dabei wurde eine Vielfalt unterschiedlicher Gratifikationsdimensionen vorgeschlagen. Hilfreiche Klassifikationsvorschläge stammen unter anderem von Katz, Hass und Gurevitch (1973, S. 166-167) oder McQuail, Blumler und Brown (1972 zitiert nach McQuail, 2010, S. 424) in Bezug auf klassische Medien, von Papcharissi und Rubin (2000, S. 186) in Bezug auf das Internet, von LaRose und Eastin (2004, S. 370) sowie Ruehl und Ingenhoff (2017, S. 178-180) im Sinne des SCU\&G in Bezug auf das Internet bzw. auf soziale Medien. Im Folgenden werden in Anlehnung an Bonfadelli und Friemel (2017, S. 77) die vier wesentlichsten Gratifikationen vorgestellt.

(1) Affektive Motive - Unterhaltung: Nach Katz et al. (1973) beziehen sich affektive Motive insbesondere auf die Suche nach ästhetischen, angenehmen 
und emotionalen Erlebnissen. Ein Hauptelement der affektiven Motive bildet die Unterhaltung (Bonfadelli \& Friemel, 2017). Der Konsum unterhaltender Medien hilft, die eigene Stimmung zu regulieren (Bonfadelli \& Friemel, 2017). McQuail et al. $(1972)^{3}$ verwenden den Begriff «Diversion» für die Flucht aus der Routine oder vor Problemen bzw. für emotionale Entspannung. In Ansätzen auf Basis der sozial-kognitiven Theorie (SC-U\&G) fallen angenehme Erlebnisse, Unterhaltung und Zeitvertreib primär in die Kategorie der Aktivitätsanreize (Ruehl \& Ingenhoff, 2017). Motive der Stimmungsregulierung oder des Zeitvertreibs werden teilweise auch der Kategorie der selbstbezogenen Anreize zugeordnet (Ruehl \& Ingenhoff, 2015a, 2017). Während bei Bonfadelli und Friemel (2017) ebenfalls den affektiven Motiven zugeordnet, bildet Eskapismus nach Katz et al. (1973) eine eigene Gratifikationskategorie. Und auch Papacharissi und Rubin (2000) unterscheiden zwischen Unterhaltungsgratifikationen und Gratifikationen in Zusammenhang mit Zeitvertreib.

(2) Kognitive Motive - Information: Kognitive Motive beziehen sich auf den Umstand, durch Medienkonsum an Informationen zu gelangen. Die gewonnenen Informationen erweitern das Wissen und das Verständnis für die Umwelt. Damit helfen sie, sich $\mathrm{zu}$ orientieren, das Leben um sich herum $\mathrm{zu}$ organisieren und es zu verstehen (Bonfadelli \& Friemel, 2017; Katz et al., 1973; Papacharissi \& Rubin, 2000). Beim SC-U\&G bilden primär Neuigkeitsanreize, aber auch selbstbezogene oder praktische Anreize das Bedürfnis nach Information ab (Ruehl \& Ingenhoff, 2015b, 2017). McQuail et al. (1972) nennen dieses Motiv «Surveillance». Surveillance handelt davon, dass Menschen über den Medienkonsum «eine Sicht auf die Ereignisse in der Welt» (McQuail, 2010, S. 572) erhalten.

(3) Sozial-interaktive Motive: Bei den sozialen Motiven oder Anreizen (LaRose \& Eastin, 2004) bzw. den «personal relationships» (McQuail et al., 1972) stehen der Kontakt zu Mitmenschen und der Umwelt sowie die Geselligkeit im Fokus (Bonfadelli \& Friemel, 2017; Katz et al., 1973; McQuail et al., 1972). Dabei spielt auch die Gruppenzugehörigkeit eine wichtige Rolle (Ruehl \& Ingenhoff, 2017). Soziale Beziehungen können durch den Medienkonsum auf vielfältige Weise profitieren, z. B. indem der Medienkonsum mit Kolleginnen und Kollegen erfolgt, indem man im Sinne einer parasozialen Interaktion eine Beziehung zu einer Person im Fernseher aufbaut, indem man sich mit anderen Menschen über rezipierte Medieninhalte austauscht oder indem man sich über Medien direkt mit anderen Menschen unterhält (Haridakis \& Hanson, 2009; Leiner et al., 2018; Palmgreen, Wenner \& Rayburn II, 1980). Der letzte Punkt hat insbesondere durch die sozialen Medien an Bedeutung gewonnen.

\footnotetext{
${ }^{3}$ McQuail et al. (2012) wird auch im Weiteren jeweils zitiert nach McQuail (2010, S. 424).
} 
(4) Integrativ-habituelle Motive: Im Zentrum integrativ-habitueller Motive steht das Verlangen nach Vertrauen, Geborgenheit oder nach Wertschätzung in verschiedenen Referenzgruppen (Bonfadelli \& Friemel, 2017). «Personal identity» nennen McQuail et al. (1972) das Motiv, wonach der Mensch Medien konsumiert, um einen Bezug zu sich selbst zu gewinnen oder die eigenen Werte zu verstärken. Im Sinne des SC-U\&G geht es bei Statusanreizen um Einfluss, Anerkennung und um die eigene Position in der Gruppenhierarchie (Ruehl \& Ingenhoff, 2017). Neben Status spielen aber auch Motive wie Stabilität, Glaubwürdigkeit und Selbstvertrauen eine Rolle (Katz et al., 1973). Bei Papacharissi und Rubin (2000) fliessen statusorientierte und soziale Motive in die Dimension der «Personal Utility» ein.

Beim SC-U\&G finden sich weiter monetäre Anreize, bei denen Medien konsumiert werden, um Geld zu sparen oder Preise zu gewinnen (Ruehl \& Ingenhoff, 2017). Dazu gehört beispielsweise die Teilnahme an einem Gewinnspiel oder Wettbewerb in sozialen Medien (Muntinga, Moorman \& Smit, 2011). Bei den ideologischen Anreizen im Sinne des SC-U\&G werden Inhalte konsumiert, weil diese die eigene Werthaltung widerspiegeln oder weil man Sympathien gegenüber dem Absender hat (Ruehl \& Ingenhoff, 2015b).

\subsection{Verwandte Ansätze}

In diesem Kapitel werden die Literaturstränge um «Advertising Value» und «Engagement» vorgestellt, weil im weiteren Verlauf der Arbeit auf die entsprechenden Arbeiten in diesen Strängen verwiesen wird. Beide Stränge berufen sich auf den U\&G-Ansatz und setzen diesen bei aktueller Forschung ein.

\subsubsection{Advertising Value}

Das Konzept des Advertising Value nach Ducoffe (1995, 1996; Ducoffe \& Curlo, 2000) wendet den U\&G-Ansatz auf Werbungen an. Ausgangspunkt des Konzepts ist die zunehmende Medienvielfalt, eine damit einhergehende erhöhte Selektivität in der Medienzuwendung und Müdigkeit Werbung gegenüber. Werbung muss unter diesen Voraussetzungen für den Rezipienten einen Nutzen haben (Ducoffe \& Curlo, 2000). Ducoffe prägte hierzu den Begriff des Werbewertes (advertising value) «as a subjective evaluation of the relative worth or utility of advertising to consumers» (Ducoffe, 1995, S. 1). Der Werbewert kann als «Index 
der Kundenzufriedenheit»(S. 1) mit der Werbung und Markenkommunikation eines Unternehmens verstanden werden (Ducoffe, 1995).

Eine zentrale Funktion von Werbung besteht demnach in ihrer Informationsleistung und der damit verbundenen Bekanntmachung von Produktalternativen. Neben der Information wird - auch hier in Bezug auf U\&G - der Unterhaltung ein positiver Effekt auf den Werbewert zugeschrieben. Bei der Informationsleistung der Werbung steht die Frage «what is said» (S. 7), bei der Unterhaltungsleistung hingen die Frage «how it is said» (S. 7) im Vordergrund (Ducoffe, 1995). Während Information und Unterhaltung einen positiven Einfluss auf den Werbewert haben, schmälert eine durch manipulative oder störende Werbetechnik hervorgerufene Irritation den Werbewert (Ducoffe, 1996).

Der Advertising-Value-Ansatz beruht auf der Annahme, dass der Werberezeption ein kommunikativer Austausch zwischen Werbetreibendem und Konsument zugrunde liegt (Ducoffe \& Curlo, 2000, S. 248). Damit ein solcher Austausch zustande kommt, muss Werbung mit einer gewissen Tiefe verarbeitet werden. Die Betrachtenden evaluieren, ob sie eine Werbung näher betrachten wollen, und bilden dabei einen «Expected Advertising Value» (EAV). Ist dieser ausreichend hoch, wird die Werbung rezipiert. Ein höherer EAV führt dabei zu mehr Involvement und zu einer stärkeren Auseinandersetzung mit der Werbung bzw. zu einer stärkeren Verarbeitungstiefe (Ducoffe \& Curlo, 2000, S. 257). Nachdem man eine Werbung gesehen hat, wird eingeschätzt, ob sich die Rezeption gelohnt hat. Dazu wird der sogenannte «Outcome Advertising Value»(OAV) gebildet (Ducoffe \& Curlo, 2000). Die Unterscheidung in (a) Erwartungen an die Rezeption und (b) die Gratifikation dieser Erwartungen durch die Rezeption orientiert sich an der oben skizzierten Unterscheidung zwischen GS und GO der U\&GForschung. OAV wiederum beeinflusst die Einstellung gegenüber der Werbung positiv.

Das Modell von Ducoffe wird nach wie vor rege genutzt, auch im digitalen Umfeld (Van-Tien Dao, Nhat Hanh Le, Ming-Sung Cheng \& Chao Chen, 2015; L. $\mathrm{Wu}, 2016)$. Allerdings bezieht sich das Konzept explizit auf Werbung und nimmt damit eine Unternehmensperspektive ein. Das zeigt sich unter anderem daran, dass es bei Information darum geht, Produktinformationen zu vermitteln. Zudem wurden vergleichbare Überlegungen schon vor Ducoffe angestellt. Kaas (1990) etwa berechnet den Nutzen der Werbung ebenfalls anhand ihres Informationsund Animationsnutzens, wobei sich Letzterer auf den Unterhaltungswert bezieht. 


\subsubsection{Engagement}

Die Erforschung des Engagements gegenüber sozialen Medien hat grosse Aufmerksamkeit erfahren. Auch dieser Literaturstrang bezieht sich stark auf den U\&G-Ansatz. Nach Khan (2017) manifestiert sich Engagement durch «an individual's interaction with media» (S. 237). Diese Interaktion kann sich auf den Konsum von Inhalten beschränken, aber auch partizipierende Handlungen (kommentieren, teilen etc.) umfassen (Khan, 2017). Es kann zwischen zwei Perspektiven unterschieden werden, wie Engagement definiert und verstanden wird (Voorveld et al., 2018, S. 39). Einer ersten, traditionellen Perspektive nach ergibt sich das Engagement anhand der Intensität und Valenz der Mediennutzung (Voorveld et al., 2018, S. 39). Das Engagement von Menschen gegenüber Medien wird dieser Perspektive nach als abhängige Variable durch Motivatoren und Einflussfaktoren zu erklären versucht. Die Motivatoren und Einflussfaktoren leiten sich teils aus der U\&G-Forschung ab. Tsai und Men (2013) untersuchen etwa das Engagement gegenüber «Brand Pages» auf Facebook anhand von sechs Motivatoren wie Unterhaltung, Information oder «soziale Integration».

Calder und Malthouse (2008, S. 21) kritisieren einen solchen verhaltensorientierten Blick auf Engagement. Medienbezogenes Verhalten (z. B. Nutzungsdauer) ist nach Ansicht der beiden Autoren eine Konsequenz und nicht Ausdruck des Engagements. Sie nehmen deshalb eine zweite Perspektive ein und schlagen eine Konzeptualisierung von Engagement basierend auf Erlebnissen (experiences) vor (Calder \& Malthouse, 2008; Calder et al., 2009; Calder, Isaac \& Malthouse, 2016). Erlebnisse sind «a consumer's beliefs about how a site fits into his/her life» (Calder et al., 2009, S. 322). Zur Definition der Erlebnisse greifen die Autoren auf die U\&G-Tradition zurück. Ein utilitaristisches Erlebnis liegt vor, wenn die Seite Informationen liefert, z. B. in Bezug auf wichtige Entscheidungen. Ein intrinsisch unterhaltsames Erlebnis hingegen hilft beispielsweise, «um dem Druck des täglichen Lebens zu entfliehen» (Calder et al., 2009, S. 322). Das Engagement selbst umschreiben sie als «the sum of the motivational experiences consumers have with the media product» (Calder \& Malthouse, 2008, S. 26). Engagement wird dieser zweiten Perspektive nach also über die Experiences gemessen bzw. bildet sich als deren Summe. Im Gegensatz zum U\&G-Ansatz können Erlebnisse auch negativer Art sein und die Abneigung gegenüber einem Medium fördern (Calder \& Malthouse, 2008, S. 40), z. B. wenn eine Zeitung zu lange Artikel produziert, die zu lesen zu viel Aufwand benötigt. Das Engagement der User gegenüber Kanälen spielt bei der Erforschung der Wirkung von Kommunikationsinhalten eine wichtige Rolle (Malthouse, Calder \& Tamhane, 2007). 
Voorveld et al. (2018) greifen die Überlegungen von Calder und Kollegen auf, weil diese es ermöglichten, unterschiedliche Medien hinsichtlich des Engagements differenziert zu vergleichen. Bei einer Messung des Engagements über Intensität und Valenz sei das nicht möglich (S. 39). Für Voorveld et al. (2018) sind Erlebnisse «the emotional, intuitive experiences or perceptions that people undergo when using a particular medium at a particular moment» (S. 40). $\mathrm{Zu}$ deren Erhebung werden Personen danach gefragt, inwiefern sie etwas Bestimmtes (z. B. «gab mir nützliche Informationen») beim Medienkonsum erlebt haben. Insofern sind Erlebnisse hier mit «Gratifications Obtained» zu vergleichen.

Dem Engagement kommt auch in Bezug auf die digitale Markenforschung (i. S. v. Brand Engagement) grosse Relevanz zu (Baldus, Voorhees \& Calantone, 2015; Calder \& Malthouse, 2008; Hollebeek, 2011; Vries, Peluso, Romani, Leeflang \& Marcati, 2017). Für Calder und Malthouse (2008) ist die Marke ein Konzept, dessen Aufgabe es ist, die Kundenerlebnisse so zu gestalten, dass die Kunden die Produkte in einer intendierten (markentreuen) Art und Weise wahrnehmen. Wie beim Engagement gegenüber Medien wird auch hier die verhaltensorientierte Dimension ausgeblendet. Im Fokus stehen die Erlebnisse mit einer Marke an den verschiedenen Kundenkontaktpunkten, die in ihrer Gesamtheit wiederum das Engagement ergeben. In Bezug auf Marken ist diese Engagement-Perspektive allerdings wenig verbreitet.

So stehen bei de Vries et al. (2017), die das Brand Engagement in sozialen Medien untersuchen, die konativen Elemente im Sinne der traditionellen Engagement-Perspektive im Vordergrund. Ihr Fokus gilt Verhaltensweisen mit hohem Engagement (z. B. Produkt-Reviews schreiben) und mittlerem Engagement (z. B. Beiträge anderer teilen) und den Einflussfaktoren, die diese Verhaltensweisen begünstigen. Die Operationalisierung der Einflussfaktoren basiert auf dem U\&G-Ansatz (Muntinga et al., 2011), aber auch auf der «Self Determination Theory» (Ryan \& Deci, 2000). Hollebeek (2011) definiert «Customer Brand Engagement» als «the level of a customer's cognitive, emotional and behavioral investment in specific brand interactions» (S. 555). Ihr Ansatz basiert ursprünglich insbesondere auf Vorarbeiten im Bereich Marketing (Vargo \& Lusch, 2004) und hat wenig Bezug zur U\&G-Forschung. In einer späteren Arbeit stehen im Sinne der U\&G-Tradition jedoch ebenfalls die Motivatoren im Fokus, die zum Customer Brand Engagement führen (Hollebeek \& Macky, 2019). Die Motivatoren oder «Erlebnisse» hinter Brand Engagement sind im Rahmen dieser Arbeit von Interesse, weil sie ebenfalls helfen, die Userperspektive des Brand Contents zu beschreiben. 


\subsection{Gratifikationen in Bezug auf Markeninhalte und soziale Medien}

Der U\&G-Ansatz kann auf verschiedene Ebenen angewendet werden und damit unterschiedliche Bezugsperspektiven einnehmen. Gratifikationen können durch den Inhalt, durch das Medium, durch den Kontext der Medienexposition, durch die Technologie oder als Prozessgratifikation durch die alleinige Interaktion mit dem Medium (z. B. zielloses Surfen im Internet) erbracht oder dort gefunden werden (N. E. Cutler \& Danowski, 1980; Katz et al., 1974, S. 514; Klapper, 1963; T. F. Stafford, Stafford \& Schkade, 2004; Sundar \& Limperos, 2013). Das Interesse gilt in dieser Arbeit drei Bezugspunkten:

1. Den Motiven, Gründen, gesuchten Gratifikationen (GS) oder Anreizen (SCU\&G) des Medienkonsums, weil dadurch die Userperspektive auf Brand Content konzeptualisiert werden kann.

2. Den durch Brand Content erhaltenen Gratifikationen (GO) bzw. deren Auswirkungen auf das Markenimage. ${ }^{4}$

3. Den Gratifikationserwartungen ${ }^{5}$ an soziale Medien, weil dadurch der Rezeptionskontext sozialer Medien beschrieben werden kann.

Die Ursprünge der Bedürfnisse, der tatsächliche Medienkonsum und Konsequenzen, die über die Befriedigung der Gratifikationen hinausgehen, sind im Rahmen dieser Arbeit sekundär. Abbildung 3.2 nach Schenk (2007, S. 686) fasst den U\&G-Ansatz zusammen und wurde um diejenigen Elemente erweitert, die im Rahmen dieser Arbeit im Fokus stehen:

\footnotetext{
${ }^{4}$ GO spielen im empirischen Teil der Arbeit eine Rolle.

${ }^{5}$ Gratifikationserwartungen verbinden Gratifikationen unter einer gegebenen Wahrscheinlichkeit mit einem spezifischen Medium oder Kanal: Wie wahrscheinlich ist es, dass ich unterhalten werde, wenn ich z. B. Instagram nutze? (siehe Abschnitt 3.5)
} 


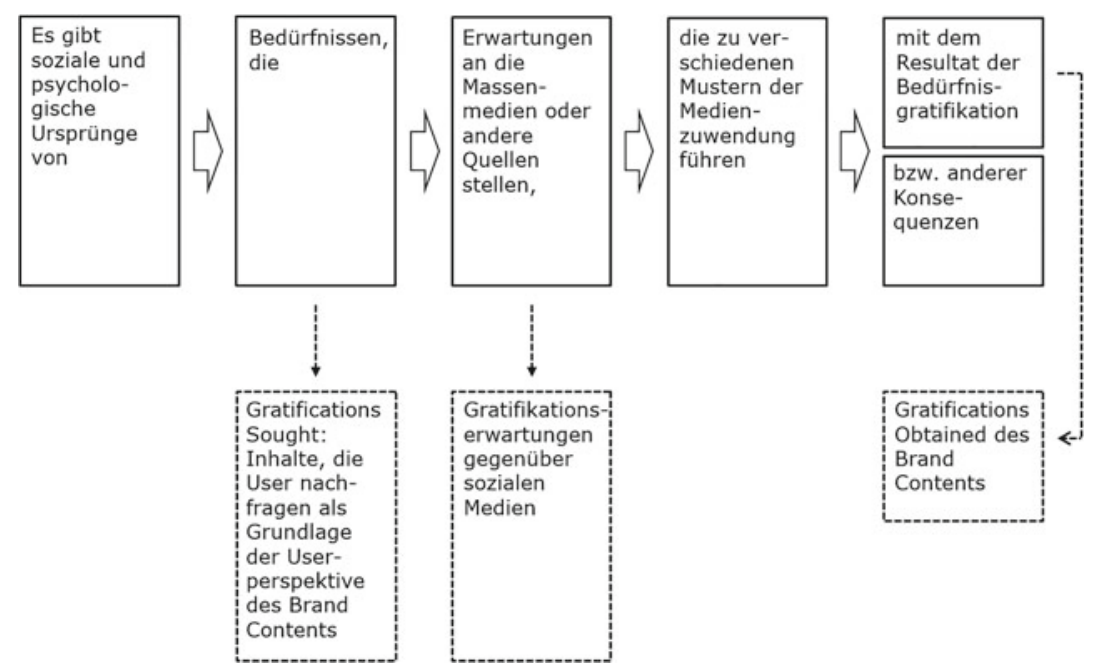

Abbildung 3.2 Elemente des Uses-and-Gratifications-Ansatzes. (Nach Schenk (2007, S. 686; volle Linien) mit eigenen Ergänzungen (gestrichelte Linien))

Durch die Interaktivität der sozialen Medien werden Gratifikationsstudien im digitalen Raum auf unterschiedliche Partizipationslevels angewendet (Shao, 2009). Es können drei Partizipationslevels unterschieden werden: Konsumieren, Partizipieren, Produzieren. Das Konsumieren repräsentiert das tiefste Interaktionslevel: Inhalte von Dritten werden passiv verarbeitet ohne weitere Eigenleistung. Auf der Stufe der Partizipation nimmt der User Bezug zu Inhalten Dritter, z. B. über das Liken, Teilen oder Bewerten von Inhalten oder durch das Verfassen eigener Kommentare. Auf der höchsten Stufe werden schliesslich eigene Inhalte (u. a. Fotos, Videos, Blog, Webseiten) produziert und digital verbreitet (Shao, 2009).

Im Folgenden werden zuerst Studien vorgestellt, die sich mit den Gratifikationen digitaler Markeninhalte beschäftigen. Dadurch soll in Erfahrung gebracht werden, aus welchen Gründen sich Menschen freiwillig mit Markeninhalten beschäftigen. Danach rücken Studien in den Fokus, welche die Gratifikationen unterschiedlicher sozialer Medien thematisieren. Dadurch soll auf die mit den Medien verbundenen Gratifikationserwartungen geschlossen werden. Da es in dieser Arbeit um die Wahrnehmung und die Wirkung von Brand Content geht, gehört 
dem Partizipationslevel «Konsum» das primäre Interesse. Die beiden Kapitel helfen auch, die in Abschnitt 3.6 vorgestellten allgemeinen Gratifikationstypologien auf ihre Aktualität zu prüfen.

\subsubsection{Gratifikationen digitaler Markeninhalte}

Die Frage, wieso User über soziale Netzwerke mit Marken interagieren, erfreut sich in der Forschung grosser Popularität. Um die Motive zu ergründen, werden Erkenntnisse der Brand-Engagement-Literatur und des U\&G-Ansatzes kombiniert. Eine grundlegende Studie hierzu legten Muntinga et al. (2011) vor. Sie untersuchten die mit den unterschiedlichen Partizipationslevels (Shao, 2009) verbundenen Gratifikationen bei 20 Internetnutzern. Bezüglich des Konsums markenbezogener Inhalte stellten sich drei Gratifikationen als wichtige Treiber heraus: Information, Unterhaltung und Belohnung (Muntinga et al., 2011, S. 26). Tsai und Men (2013) untersuchten unter Verweis auf Muntinga et al. (2011), aus welchen Motiven 280 US-amerikanische Facebook-Nutzer mit Marken über Facebook interagieren. Sie fassen die Ergebnisse folgendermassen zusammen: «The respondents typically used a Facebook brand page as a platform to search for discount or sales news, to exchange information with other members, and to have fun and seek leisure» (Tsai \& Men, 2013, S. 84). Die Ergebnisse ihrer Studie decken sich demnach mit denjenigen von Muntinga et al. (2011).

Das Interesse von Enginkaya und Yilmaz (2014) gilt den Motivationen, aus welchen sich 493 Studierende über Social Media mit Marken austauschen bzw. mit ihnen interagieren. Traditionelle Gratifikationen werden mit Erkenntnissen aus der Markenliteratur ergänzt. Die Autoren identifizierten fünf Faktoren: Markenzugehörigkeit, Investigation, Suche nach «Opportunities», Konversation und Unterhaltung. Bei Investigation steht die Suche nach Produktinformationen im Vordergrund. «Opportunity Seeking» birgt grosse Ähnlichkeiten zum Konstrukt

Belohnung bei Muntinga et al. (2011). Es geht darum, über Promotionen, Rabatte oder neue Angebote informiert zu werden bzw. davon profitieren zu können (Enginkaya \& Yılmaz, 2014).

Ruehl und Ingenhoff (2015a) wenden den SC-U\&G-Ansatz an, um herauszufinden, aus welchen Gründen Politiker und Digital Natives über soziale Netzwerke mit Unternehmen interagieren. 65 semistrukturierte Interviews mit Repräsentierenden der beiden Zielgruppen zeigen auf, dass diese die Inhalte auf den Unternehmensprofilen hauptsächlich konsumieren, um Informationen über die Unternehmen und deren Produkte und Leistungen zu bekommen. Politiker interagieren dabei vorzugsweise über Twitter und Digital Natives über Facebook 
mit Unternehmen (Ruehl \& Ingenhoff, 2015a, S. 294). Mittels einer OnlineUmfrage unter 215 Facebook-Nutzern vertieften die beiden Forschenden in einer zweiten Untersuchung die Nutzungsmotive hinsichtlich Facebook Brand Pages (Ruehl \& Ingenhoff, 2017). Aktivitätsmotive (Unterhaltung, Spass, Zeitvertreib), selbstbezogene Anreize im Sinne einer Meinungsbildung (über Produkte, Services, das Unternehmen allgemein) und monetäre Motive (Teilnahme an Wettbewerben, Rabatte, Teilnahme an Spielen) konnten als die wichtigsten Treiber hinter dem Konsum von Inhalten eruiert werden (Ruehl \& Ingenhoff, 2017, S. 179).

Pöyry, Parvinen und Malmivaara (2013, S. 226) machten utilitaristische und hedonistische Motive als die grundlegenden Treiber hinter der Teilnahme an der Facebook Brand Community einer europäischen Reise-Agentur unter 1'162 Community-Mitgliedern aus. Utilitaristische Motive sind dabei mit einem Ziel verbunden, z. B. bestimmte Informationen zu finden. Bei hedonistischen Motiven geht es hingegen darum, Spass oder Unterhaltung zu erfahren.

Athwal, Istanbulluoglu und McCormack (2019) ermittelten die Gratifikationen, auf Basis welcher Jugendliche Luxusmarken auf Facebook, Twitter oder Instagram folgen. Dazu führten sie qualitative Gespräche mit 30 Personen mit vorwiegend britischer Nationalität. Die Gespräche offenbarten kognitive und affektive Motive als die primären Treiber. Apaolaza, Hartmann, He, Barrutia und Echebarria (2017) untersuchten, wieso chinesische Jugendliche auf QZone mit Marken interagieren. Sie konnten einen positiven Einfluss der auf QZone erwarteten Unterhaltung, jedoch keinen Einfluss von Socializing oder Information auf die Interaktion mit Marken nachweisen.

Sook Kwon, Kim, Sung und Yun Yoo (2014) interessierten sich für die Gründe, weshalb Marken auf Twitter gefolgt wird. Auf Basis einer Umfrage unter 400 «Brand Followers» konnten sie vier primäre Gründe eruieren. Bei Incentives geht es darum, Vorteile zu erlangen, z. B. in Form von exklusiven Inhalten, Rabatten oder Spezialangeboten. Insofern ist diese Kategorie vergleichbar mit monetären Motiven des SC-U\&G (Ruehl \& Ingenhoff, 2015a). Hinter den sozial-interaktiven Motiven steht sowohl das Bedürfnis, sich selbst Gehör zu verschaffen, als auch die Meinung anderer bezüglich der Marke zu erfahren. Information umschreibt das Bedürfnis, über Neuigkeiten der Marke informiert zu werden. Das Motiv Brand Usage/Liking umschreibt die Tatsache, dass die Follower die Marke nutzen oder schlicht mögen (Sook Kwon et al., 2014).

Die Resultate von Piehler, Schade, Kleine-Kalmer und Burmann (2019) zeigen, dass 359 befragte Facebook-User Markeninhalte auf Facebook primär zu Unterhaltungszwecken und aus Gründen der sozialen Interaktion konsumieren. Der Zugang zu Informationen als Motivator konnte in ihrer Studie nicht nachgewiesen werden. 


\subsubsection{Gratifikationserwartungen an soziale Medien}

Lev-on (2017) stellt in einer Metaanalyse über publizierte U\&G-Artikel eine rege Forschungstätigkeit rund um die digitale Kommunikation fest. Sie macht bei der internetbezogenen U\&G-Forschung bisher drei Entwicklungsschritte aus: Mit dem Aufkommen des Internets in den 1990er-Jahren begannen sich Forscher mit den Gratifikationen dieses neuen Mediums auseinanderzusetzen. Das Internet wurde dabei meist als ein Medium wahrgenommen (S. 2013). Mitte der 2000er-Jahre verschob sich der Fokus auf die aufstrebenden sozialen Netzwerke und die damit verbundenen usergenerierten Inhalte. Während sich Studien zuerst wieder einzelnen sozialen Netzwerken in ihrer Gesamtheit widmeten (z. B. die Gratifikationen von Facebook), wurde auch hier der Untersuchungsgegenstand zunehmend fragmentiert und einzelne Aspekte von sozialen Netzwerken (z. B. Communitys oder Gruppen) rückten in den Fokus (Lev-on, 2017). Der dritte Entwicklungsschritt setzte ab Mitte der 2010er-Jahre ein und dauert an. Im Zentrum dieses dritten Schrittes stehen das Smartphone, die mobile Kommunikation und zunehmend auch Aspekte des «Internet of Things» (Lev-on, 2017, S. 2009-2010).

Im Folgenden stehen insbesondere Studien des zweiten Entwicklungsschrittes im Fokus. Es soll herausgearbeitet werden, welche sozialen Medien mit welcher Art von Gratifikationen verbunden werden und welche Gratifikationserwartungen sich aus Usersicht als Konsequenz daraus ergeben. W. Schweiger (2007) spricht in diesem Zusammenhang von einer medienzentrierten Sichtweise des U\&G-Ansatzes, bei welcher Bedürfnisbefriedigung «als Medienfunktion» (S. 66) verstanden werden kann. Bei Schenk (2007) findet sich hierzu der Begriff der «Gratifikationsleistung der Medien» (S. 711). Flanagin und Metzger wiederum sprechen von funktionalen Images, die sich auf Basis der Nutzung und der Funktionen pro jeweiligem Medium bilden:

[...] functional images [Hervorhebung im Original] of media distinguish communication technologies according to their most salient features and uses (i. e., functions). The functional image of a medium is based on collectively held notions of how the medium is used that are formed through its long-term use. (Flanagin \& Metzger, 2001, S. 159)

Nach Lichtenstein und Rosenfeld kann davon ausgegangen werden, dass das «Image der Medien» - welche Gratifikationen befriedigen sie besonders gut? unabhängig vom Nutzungsumfang und von der Einstellung gegenüber dem Medium ist: «Each medium appears to possess an image independent of its use» 
(Lichtenstein \& Rosenfeld, 1983, S. 107). In dieser Arbeit wird - in Anlehnung an SC-U\&G - von Gratifikationserwartungen gegenüber sozialen Medien gesprochen. Wie in Abschnitt 3.5 hergeleitet, beschreiben Gratifikationserwartungen die Wahrscheinlichkeit, eine bestimmte Gratifikation durch ein spezifisches Medium oder ein spezifisches medienbezogenes Verhalten zu bekommen. Damit sind Gratifikationserwartungen gewissermassen Gratifikationen (i. S. v. Motiven, GS, Bedürfnissen), die sich mit einer gewissen Wahrscheinlichkeit bei einem bestimmten Medium finden. Deshalb werden im Folgenden die mit sozialen Medien typischerweise verbundenen Gratifikationen vorgestellt, um dadurch auf die Gratifikationserwartungen zu schliessen. Dabei werden diejenigen Kanäle vorgestellt, die für Schweizer Unternehmen und User relevant sind (Koob, 2018; Y\&R Group Switzerland, 2018) und die als mögliche Kanäle in der empirischen Studie dieser Arbeit in Frage kommen.

\section{Gratifikationen Facebook}

Die wissenschaftliche Auseinandersetzung mit den Gratifikationen von Facebook begann früh nach der Gründung der Plattform im Jahr 2004. Die ersten Studien zeigen, dass Facebook primär dazu genutzt wird, um soziale Kontakte zu pflegen und mit bestehenden und alten Freunden in Kontakt zu bleiben. Die Suche nach Informationen über Kollegen und neue Bekanntschaften spielt ab Beginn eine zentrale Rolle (Joinson, 2008; Lampe, Ellison \& Steinfield, 2006; Raacke \& Bonds-Raacke, 2008). Seither haben sich etliche weitere Studien mit den Gründen der Facebook-Nutzung auseinandergesetzt. Die Bedeutung der sozialen Interaktion wurde dabei wiederholt bestätigt, zudem wurden Gratifikationen wie Unterhaltung, "Lifeblogging», Mitteilungsbedürfnis, Zeitvertreib oder Information gefunden (Giannakos, Chorianopoulos, Giotopoulos \& Vlamos, 2013; Karapanos, Teixeira \& Gouveia, 2016; Smock, Ellison, Lampe \& Wohn, 2011). Auch Leiner et al. (2018, S. 200) stellten unter 482 Mitgliedern eines Online-Panels aus Deutschland, der Schweiz und Österreich fest, dass der Konsum von Inhalten auf Facebook durch vielfältige - kognitive, affektive, soziale, persönliche, eskapistische - Gratifikationen erklärt wird.

\section{Gratifikationen Twitter}

Mit den Twitter-Gratifikationen beschäftigen sich die Arbeiten von G. M. Chen (2011) und Johnson und Yang (2009). Johnson und Yang machen sowohl als gesuchte (GS) als auch als erhaltene Gratifikationen (GO) soziale und informative Motive unter 242 Twitter-Nutzern aus. Unter den sozialen Motiven subsumieren sie unter anderem Zeitvertreib, Unterhaltung, Spass, Entspannung, sich ausdrücken oder mitbekommen, was andere tun. $\mathrm{Zu}$ den informativen Motiven 
gehören die Informationsgewinnung, Rat geben und erhalten oder neue Leute kennenlernen (Johnson \& Yang, 2009, S. 17). Indem sie die gesuchten den erhaltenen Gratifikationen gegenüberstellten, konnten sie den Grad der Zufriedenheit mit der Twitter-Nutzung messen. Es zeigt sich, dass Twitter einzig in Bezug auf «Spass haben» die gesuchten Gratifikationen nicht befriedigen konnte. G. M. Chen (2011) zeigte anhand einer Befragung von 317 Twitter-Nutzern, dass mit zunehmender Nutzung von Twitter soziale Gratifikationen besser befriedigt werden.

Im Vergleich zu Facebook-Nutzern sind Twitter-Nutzer intellektuell und kreativ offener und interessieren sich insbesondere für Inhalte und Neuigkeiten über intellektuelle und kreative Aktivitäten (Marshall, Ferenczi, Lefringhausen, Hill \& Deng, 2018). Die Autoren konnten allerdings unter den britischen Studienteilnehmern keinen positiven Einfluss von Informationsmotiven auf die Twitter-Nutzung nachweisen (Marshall et al., 2018).

\section{Gratifikationen Instagram}

Den Gründen der Instagram-Nutzung gehen Sheldon und Bryant (2016) nach. Mittels einer Fokusgruppe und anschliessender Umfrage unter 239 amerikanischen College-Studierenden konnten sie vier Hauptmotive beschreiben: (1) Überwachung und Wissen über andere, (2) Dokumentation, (3) Coolness und (4) Kreativität. Die Überwachungs- und Wissensfunktion stellte sich als der stärkste Treiber der Instagram-Nutzung heraus. Die Autoren sehen darin eine Parallele zum Motiv der Informationssuche (Sheldon \& Bryant, 2016, S. 94). Ko und Yu (2019) machten unter 328 taiwanesischen Instagram-Usern Unterhaltung, Zeitvertreib, das Aufrechterhalten sozialer Kontakte und Information als Gründe des Konsums von Videos im Story-Format aus.

\section{Gratifikationen YouTube}

Informationssuche, Unterhaltung, "Co-Viewing» und soziale Interaktionen stellten sich als signifikante Prädikatoren des Videokonsums bei 427 Studierenden heraus (Haridakis \& Hanson, 2009). Die drei letzteren Motivationen hatten zudem einen positiven Einfluss auf das Teilen von YouTube-Videos (Haridakis \& Hanson, 2009). Mittels einer Umfrage unter 1'143 registrierten YouTube-Usern gelangte Kahn (2017) zum Ergebnis, dass YouTube-Videos primär zu Unterhaltungszwecken und die Kommentare zu den Videos aus Informationszwecken konsumiert werden. Mittels einer Umfrage unter 101 taiwanesischen HighSchool-Studierenden zeigten Yen, Chang und Chou (2019), dass die Nutzung von Corporate-Finance-Lernvideos primär durch die Gratifikationen Unterhaltung und Information, aber auch durch «Convenience» erklärt wird. 


\section{Gratifikationen Snapchat}

Analog zu Instagram werden über Snapchat primär Bilder und Videos ausgetauscht. In einer Studie unter 274 Snapchat-Nutzern konnten Pittman und Reich (2016) zeigen, dass dieser bildbasierte Austausch Intimität fördert, Einsamkeit reduziert und daher insbesondere auf sozialen Motiven gründet (S. 164). Eine Studie unter 77 primär schottischen und britischen Facebook- und SnapchatUsern ergab, dass diese Snapchat für Prokrastination benutzen, aber auch, um in Kontakt mit Familie und Freunden zu bleiben sowie um zu sehen, was andere Personen machen. Zudem wird Snapchat im Vergleich zu Facebook stärker zum «Flirten» gebraucht (Utz, Muscanell \& Khalid, 2015). Piwek und Joinson (2016) befragten 209 ebenfalls englische Studierende zu ihrer Snapchat-Nutzung. Ihre Resultate zeigen, dass die User über Snapchat im Vergleich zu Facebook mit einer kleineren Zahl an anderen Nutzern interagieren. Snapchat wird daher stärker für «Bonding» (enge Beziehungen pflegen, z. B. mit Familie) als für «Bridging» (lose Kontakte pflegen, z. B. mit ehemaligen Schul- oder Arbeitskollegen) benutzt (S. 364). Spinda und Puckette (2018) untersuchten, aus welchen Motiven Sportinhalte auf Snapchat rezipiert werden. In einem zweistufigen, qualitativen und quantitativen Vorgehen unter 312 Personen identifizierten sie vier Hauptmotive: «ease and convenience», «behind the scenes», «vicarious experience» und «unique points of view».

Durch unterschiedliche Betitelungen und Operationalisierung der Gratifikationen, durch unterschiedliche empirische Ansätze und durch unterschiedliche Stichproben ist es schwierig, auf Basis der genannten Partikularstudien auf die Gratifikationserwartungen einzelner sozialer Medien gegenüber anderen sozialen Medien zu schliessen. Zu einem ähnlichen Fazit kommt auch M. Krcmar (2017, S. 2004): «As such, most Internet studies that have examined specific web activities have presented their own typologies of uses and gratifications.» Abhilfe kann hier allerdings eine medienvergleichende Studie schaffen.

\section{Gratifikationen soziale Medien - medienvergleichend}

Voorveld et al. (2018) bedienen sich des Engagement-Konstrukt, das sie in Anlehnung an Calder, Isaac und Malthouse (2016) in verschiedene Erlebnisse (experiences) aufteilen. Diese Erlebnisse decken in der Tradition des U\&GAnsatzes unterschiedliche Gratifikationen ab. Die Autoren untersuchten die Erlebnisse von acht sozialen Medien (Facebook, Twitter, YouTube, LinkedIn, Google + , Snapchat, Instagram und Pinterest) unter 1'919 Internetnutzern in den Niederlanden. Ihre Resultate zeigen, dass die sozialen Medien unterschiedliche Erlebnisse und damit ein unterschiedliches Engagement verursachen. Facebook wird insbesondere für soziale Interaktionen, zu Informationszwecken (Topicality) 
und für den Zeitvertreib genutzt. Twitter sorgt dafür, dass Nutzer rasch informiert sind und up-to-date bleiben. Auf weiteren Dimensionen erreichte Twitter vergleichsweise tiefe Erlebnis-Werte. YouTube dient der Unterhaltung, während LinkedIn in erster Linie mit Information in Verbindung gebracht wird. Das Engagement gegenüber Snapchat besteht hauptsächlich aus Unterhaltungserlebnissen und Erlebnissen rund um soziale Interaktionen. Instagram erreichte die höchsten Werte bei Topicality und Zeitvertreib, wird aber auch stark zur Unterhaltung und sozialen Interaktion genützt. Mit Pinterest verbinden die Befragten «Stimulation», das heisst, dass die Inhalte auf Pinterest originell und neu sind. Die Autoren (Voorveld et al., 2018) kommen zum Schluss, dass «each digital platform is experienced in a unique way» (S. 50).

Auf Basis der Studie von Voorveld et al. (2018) lässt sich erahnen, dass Twitter tendenziell mit Informationserwartungen und Plattformen wie YouTube, Instagram oder Facebook primär mit Unterhaltungserwartungen verbunden werden. Weil diese Unterscheidung aber nur in dieser Studie präzise zum Ausdruck kommt, werden die Gratifikationserwartungen dreier sozialer Medien im empirischen Teil der Arbeit für die Schweiz im Detail untersucht (siehe Abschnitt 6.5.1).

\subsection{Zwei zentrale Gratifikationen im Fokus}

In den obigen Kapiteln wurde dargestellt, dass der Konsum von Medien und Markeninhalten sowie die Nutzung sozialer Medien durch unterschiedliche Gratifikationen erklärt werden kann. Für W. Schweiger (2007, S. 61) sind das Informations- und das Unterhaltungsbedürfnis die beiden wichtigsten Bedürfnisse. Die Konzeptualisierung von Medieninhalten auf dem Kontinuum Unterhaltung versus Information hat entsprechend eine lange Tradition. W. Schramm (1949) präsentiert in seiner Analyse zur Zeitungsnutzung einen «immediate spleasure> reward» und einen «delayed 〈reality〉 reward» (S. 268). Ersterer dreht sich um das Vergnügen, die Unterhaltung und die Erholung während des Lesens, Letzterer dreht sich um die relevante Informationsgewinnung (W. Schramm, 1949, S. 261). Auch Weiss (1971) fasst später zusammen, dass U\&G-Studien Gratifikationen oft dichotom als «fantasy-escapist» oder «informational-educational» (S. 312) ansehen. Was in Bezug auf traditionelle Medien herausgefunden wurde, konnte später für das Internet bestätigt werden: «Among the main reasons for using the Internet are finding useful information and browsing for entertainment» (Schlosser, 2003, S. 185). In Deutschland werden in einer Langzeitstudie alle fünf Jahre «die Funktionen und Images der Medien» verglichen (Breunig \& Engel, 2015). Die drei Hauptgründe der Internetnutzung sind: (1) sich informieren, (2) Nützliches 
für den Alltag erfahren, (3) macht Spass. Es zeigte sich weiter, dass Information und Unterhaltung für den passiven Medienkonsum in sozialen Medien die relevantesten Gratifikationen sind (Shao, 2009, S. 15). Und beim Konsum markenbezogener Inhalte erwarten Userinnen und User neben «monetären» Vorteilen und sozialen Aspekten ebenfalls primär Information und Unterhaltung (Muntinga et al., 2011; Ruehl \& Ingenhoff, 2017; Taiminen \& Karjaluoto, 2017). Hollebeek und Macky (2019) machen funktionale und hedonistische Motive gar als die beiden «generischen» (S. 32) Usermotive hinter Digital Content Marketing aus.

Im Rahmen dieser Arbeit wird entsprechend auf Information und Unterhaltung fokussiert; einerseits, um die Userperspektive des Brand Contents zu konzeptualisieren, andererseits, um die Gratifikationserwartungen an soziale Kanäle zu bestimmen.

Obwohl sich die beiden Dimensionen fast durchgehend in allen U\&G-Studien finden, werden diese oftmals nur rudimentär beschrieben und dann anhand von Items operationalisiert (z. B. Piehler et al., 2019). Insbesondere, um die Userperspektive des Brand Contents zu definieren und um Brand-Content-Botschaften für den empirischen Teil zu entwickeln, ist ein inhaltliches Verständnis der beiden Gratifikationen unerlässlich. Im Folgenden folgt deshalb eine Detailbetrachtung der Konzepte Information und Unterhaltung.

\subsubsection{Information}

Information ist ein vielfältiger Begriff, der in unterschiedlichsten Wissenschaften divergent verstanden und angewandt wird (siehe Ott, 2004, S. 15). Ansätze zur Definition von Information, wie sie für diese Studie hilfreich sind, finden sich insbesondere in der Kommunikations- (Bosshart, 2007) und in der Informationswissenschaft (Buckland, 1991). Beiderorts wird Information in einen Kommunikationskontext eingebettet und in ein Verhältnis zu Begriffen wie Wissen (knowledge), Daten (data), Botschaft (message) oder Effekt (impact) gesetzt (Meadow \& Yuan, 1997).

Es kann zwischen einem objektiven und einem subjektiv-situativen Verständnis von Information unterschieden werden (Hjørland, 2007). Information liegt dem objektiven Verständnis nach beim Objekt selbst, besteht unabhängig davon, ob jemand informiert wird oder nicht, und ist nicht mit dem Prozess des «Informierens» vereinbar (Hjørland, 2007, S. 1450). Die objektive Sichtweise geht 
vom Ansatz «any difference is information» (S. 1449) aus (Hjørland, 2007). Insbesondere technisch orientierte Kommunikationstheorien (z. B. Shannon, 1948) bedienen sich des objektiven Informationsbegriffs (Buckland, 1991, S. 359; Burkart, 2019, S. 370).

Gemäss dem subjektiven Verständnis wird Information nicht übertragen, sondern entsteht beim Rezipierenden auf Basis der im Kommunikationsprozess übertragenen Signale (Beck, 2017, S. 21). Information kann mit Verweis auf den lateinischen Ursprung «informare» als ein Prozess verstanden werden, bei dem es unter anderem darum geht, etwas zu formen oder ein Bild von etwas zu entwerfen (Beck, 2017, S. 23). Jeder Mensch verarbeitet, interpretiert und nutzt erhaltene Signale unterschiedlich. Derselbe Zeitungsartikel kann bei unterschiedlichen Lesern zu unterschiedlichen Informationen führen. Bei Information handelt es sich also um «das Ergebnis eines individuellen Konstruktionsprozesses» (Beck, 2017, S. 54). Was in einer Situation A oder für eine Person A Information ist, muss in einer Situation B oder für eine Person B keine Information sein. Information ist damit im Sinne des subjektiven Begriffsverständnisses immer Interpretationssache des Rezipierenden (Hjørland, 2007, S. 1449).

Information kann zu Wissen werden (H. Krcmar, 2015, S. 12). Wissen ist jedoch auch Ursprung von Information. Auf die zweiseitige Beziehung von Information und Wissen weisen Schmid und Lyczek (2008) hin: «Wissen kann deshalb Ergebnis der Kommunikation von Information sein. Wissen ist aber auch die Quelle von Information: Information stellt symbolisiertes (externalisiertes) Wissen dar» (S. 13).

Bateson (1972) legte mit seiner Aussage, Information sei «any difference which makes a difference in some later event» (S. 381), die Grundlage für das subjektive Verständnis von Information (Hjørland, 2007, S. 1449). Sein Begriffsverständnis veranschaulicht Bateson (1972, S. 315-316) anhand der Funktionsweise einer Dampfmaschine: Teilt die Maschine einem Kontrollmechanismus oder Regulator (governor) den Unterschied zwischen der intendierten und der optimalen Laufgeschwindigkeit mit (Differenz 1) und unternimmt der Regulator aufgrund dieser Botschaft Massnahmen, z. B. im Sinne einer Beschleunigung oder Bremsung (Differenz 2), kann die Mitteilung als Information klassifiziert werden. Information muss also nicht nur im Sinne von Neuartigkeit das Wissen des Rezipienten mehren, sondern ebenfalls im Sinne von Effektivität zukünftige Handlungen beeinflussen. Meadow und Yuan (1997) sprechen in ähnlicher Weise vom Effekt der Information. Nach ihnen kann dieser Effekt aber auch «nur» darin bestehen, die Wissensstruktur des Empfängers zu ändern (S. 700). Oftmals hat aber die geänderte Wissensstruktur ihrerseits Effekte auf zukünftige Entscheidungen oder Handlungen: 
«It [the input signal] changes the knowledge state and the new knowledge state triggers the delivery of the decision» (Meadow \& Yuan, 1997, S. 703).

Bosshart (2007) orientiert sich bei seiner Definition von Information an Bateson (1972) und Ott (2004). Information besteht demnach aus drei grundlegenden Elementen: «Neuigkeit, Relevanz und Richtigkeit» (Bosshart, 2007, S. 17). Neuigkeit bedeutet, dass Information Wissen erweitert und Unsicherheit reduziert, Relevanz bezieht sich auf die Nützlichkeit der Information für unser Leben (Bosshart \& Hellmüller, 2009, S. 6). Information gibt Antworten auf Fragen, schreibt Ritchie (1991, S. 3) diesbezüglich. Wittmann (1959) meint: «Information ist zweckorientiertes Wissen [Hervorhebung im Original], also solches Wissen, das zur Erreichung eines Zweckes (...) eingesetzt wird» (S. 14). Nützlichkeit ist schliesslich nur gegeben, wenn die Information korrekt ist (Bosshart \& Hellmüller, 2009, S. 6). Dieser Punkt ist allerdings umstritten (Buckland, 1991, S. 356). H. Krcmar (2015) spricht nicht vom Gegensatz korrekt - inkorrekt, sondern verweist darauf, dass Information in unterschiedlicher Qualität hinsichtlich ihrer «Genauigkeit, Vollständigkeit und Zuverlässigkeit» (S. 17) vorliegen kann. Auch Meadow und Yuan (1997) sprechen einzig davon, dass Symbole «verständlich, neu oder bedeutungsvoll» (S. 704) sein müssen, um als Information klassifiziert zu werden.

Buckland (1991) unterscheidet «information-as-process», «information-asknowledge» und «information-as-thing». Information-as-thing kann als ein mit «Information» angereichertes Objekt umschrieben werden. Buckland (1991) versteht darunter «objects, such as data and documents, that are referred to as 〈information〉 because they are regarded as being informative» (S. 351). Primär kann alles «Information» sein, solange der Mensch sich deren Existenz (bzw. der Existenz des Objekts) gewahr ist (S. 356). Information-as-process impliziert, dass ein Mensch «informiert» wird, was sich in neuem oder erweitertem Wissen (information-as-knowledge) manifestiert (Buckland, 1991, S. 352). Obwohl prinzipiell alle Objekte «Information» sein können (information-as-thing), ergeben sich information-as-process und information-as-knowledge in Abhängigkeit von Person und Situation. Nur wenn diese beiden Prozesse aktiviert werden, wird das Objekt in dieser Situation und bei dieser Person informativ und zum information-as-thing. Weil bei gewissen Objekten aber mit hoher Wahrscheinlichkeit davon ausgegangen werden kann, dass diese in der Zukunft in einer spezifischen Situation «informativ» sein werden, können diese als «Information» tituliert werden (S. 357). Während bei Buckland information-as-thing den objektiven Informationsbegriff abdeckt, nehmen Information-as-process und information-as-knowledge ein subjektives Begriffsverständnis ein. 
Für diese Arbeit bleibt Information dem subjektiven Wissensgewinn vorbehalten, während informativer (i. S. v. Mehrwert aus Userperspektive) Brand Content über «Informationspotenzial» verfügt, d. h., dass dieser im Sinne von Bucklands (1991) information-as-thing mit hoher Wahrscheinlichkeit das Wissen des Lesers oder der Leserin erweitert, deren Unsicherheit reduziert und einen positiven Effekt auf deren zukünftige Handlungen hat.

\subsubsection{Unterhaltung}

Für Bosshart (2007, S. 18) ist Unterhaltung primär ein Rezeptionsphänomen, das nicht durch das Unterhaltungsangebot oder -produkt determiniert ist (Bosshart \& Hellmüller, 2009, S. 3), sondern als Erlebnis auf der Seite des Rezipierenden entsteht. Unterhaltung kann dabei verschiedene Formen annehmen, wie Entspannung, Abwechslung und Zeitvertreib, Anregung und Stimulation, Spass, Atmosphäre oder Freude (Bosshart \& Macconi, 1998, S. 4). Verdichtet kann Unterhaltung anhand dreier primärer Komponenten charakterisiert werden: Unterhaltung ist aktiv, positiv und baut Spannung ab (Bosshart \& Macconi, 1998, S. 4).

Unabhängig von Form und Ausprägung geht es bei Unterhaltung letztlich um das Erleben von Vergnügen: «We define entertainment, in its broadest sense, as any situation or activity from which a person derives pleasure» (Bosshart \& Hellmüller, 2009, S. 3). Das persönliche Vergnügen kann sich dabei auf Körper, Sinne, Gefühle, Intellekt, Witz oder Mitgefühl beziehen (Bosshart, 2006, S. 17).

Nach Vorderer und Reinecke (2015) basiert diese Sichtweise auf einem hedonistischen Konsumentenbild, wonach Unterhaltung der Befriedigung hedonistischer Bedürfnisse und der Regulierung der eigenen Stimmung dient. Letztere Erkenntnis fand insbesondere in der und durch die Mood-Management-Theorie von Zillmann (1988) Beachtung. Der Theorie liegt die Annahme zugrunde, dass der Konsum unterhaltender Medieninhalte den Menschen hilft, die eigene Stimmung zu beeinflussen. Konsumenten wenden sich entsprechend Medieninhalten zu, die ihnen helfen, eine positive Stimmung zu erlangen, diese zu verstärken oder zu verlängern. Sie meiden hingegen Inhalte, die eine negative Stimmung erzeugen, verstärken oder verlängern (Zillmann, 1988). Die Auswahl kann bewusst oder unbewusst («feel like doing this or that», S. 329) geschehen, wird allerdings stark durch frühere Erlebnisse und Erfahrungen beeinflusst (Zillmann, 1988). Eine rein hedonistische Perspektive auf das Unterhaltungserlebnis sei, so Vorderer und Reinecke (2015), schwer vereinbar mit Unterhaltungsangeboten, die den User in eine traurige Stimmung versetzen (z. B. ein Drama). Um diese Diskrepanz zu 
erklären, ist Unterhaltung als Metaemotion konzipiert worden, die sich auf einem übergeordneten Level bildet, selbst wenn das Unterhaltungsangebot Primärreaktionen wie Angst oder Traurigkeit verursacht (Vorderer \& Reinecke, 2015). Die «triadisch-dynamische Unterhaltungstheorie TDU» ist ein solcher Ansatz, der Unterhaltung als Metaemotion versteht. Der Theorie liegen zwei Prämissen zugrunde (Früh \& Wünsch, 2007):

- Unterhaltung ist ein Rezeptionserlebnis: Weil Unterhaltung als Erlebnis aufseiten des Rezipienten entsteht, produzieren Medien «nicht Unterhaltung, sondern nur Unterhaltungsangebote» (Früh \& Wünsch, 2007, S. 33).

- Unterhaltung ist ein positives Erlebnis.

Damit Unterhaltung von anderen positiven Erlebnissen - Früh und Wünsch (2007) nennen als Beispiele die Freude nach einer erfolgreichen Prüfung oder nach einem Olympiasieg - abgegrenzt werden kann, ist eine dritte Annahme erforderlich, nämlich die der «Souveränität und Kontrolle». Erst Souveränität und Kontrolle, so Früh (2006), kreieren, was als «charakteristischer Akzent» (S. 40) des Unterhaltungserlebens bezeichnet werden kann. Kontrolle bedeutet, dass der Rezipient die Konsequenzen des Unterhaltungskonsums überschauen und beherrschen kann. Mit Souveränität ist die «Dispositions- und Entscheidungsfreiheit der Rezipienten» (S. 40) gemeint (Früh, 2006). Das bedeutet, dass Unterhaltung freiwillig konsumiert wird und der Konsum jederzeit beendet werden kann (W. Schweiger, 2007, S. 219).

In ähnlicher Weise umschreibt Hügel (2007) dieses der Unterhaltung zugrundeliegende Phänomen, wonach Unterhaltung «(fast) ernstgenommen und (fast) bedeutungslos zugleich sein [will]» (S. 25), als ästhetische Zweideutigkeit:

Wir sind, während wir uns unterhalten, mit Geist, Seele und Leib anwesend, setzen uns nur nicht dem Druck aus, auf das Erlebte sofort psychisch, seelisch oder handelnd reagieren zu müssen. Unterhaltend behalten wir, die Rezipienten, den Umfang unserer Reaktion in der Hand. Weder werden wir gebannt von großer Mimen großer Kunst noch, wie von einem politischen Redner, ernstlich gefangengenommen, zu einer Reaktion, ja zu Handlungen gedrängt; noch lässt uns das, was wir unterhaltend erleben, völlig kalt. (Hügel, 2007, S. 25)

Unterhaltung entsteht bei Früh im Zusammenspiel zwischen der Person, dem Stimulus und dem situativen, gesellschaftlichen Kontext. Nur wenn sich die drei Variablen ergänzen («triadisches Fitting»), kann Unterhaltung als «kognitivaffektives Erleben auf der Makroebene» (Früh \& Wünsch, 2007, S. 41) entstehen. 
Der Rezeption der Inhalte - und damit auch der Entstehung von Unterhaltung - liegt ein steter Informationsverarbeitungsprozess zugrunde. Inhalte, Szenen oder Ausschnitte werden kontinuierlich analysiert und evaluiert. Die Wahrnehmung einzelner Szenen, Inhalte oder Ausschnitte führt zu Mikroemotionen, die zu einem Gesamturteil auf der Makroebene zusammenfliessen. Dadurch ist es auch möglich, dass negative Mikroemotionen wie Angst oder Trauer (z. B. in einem Thriller oder Drama) zu einem positiven Unterhaltungserleben führen (Früh \& Wünsch, 2007). Untersuchungen zeigten beispielsweise, dass Mitgefühl gegenüber Protagonisten in einer tragischen Geschichte in positiven Gefühlen resultieren kann (Wied, Zillmann \& Ordman, 1994).

Dieser Erklärungsumweg über Metaemotionen, so Vorderer und Reinecke (2015), ändere jedoch nichts an der Auffassung, wonach Unterhaltung eine hedonistische Gratifikation ist, unabhängig davon, ob die Unterhaltung hedonistisch (Komödie) oder nicht-hedonistisch (Drama) ist. Diese enge Sichtweise auf den Unterhaltungsbegriff, wonach Unterhaltung allein der Befriedigung hedonistischer Motive dient, wird zunehmend kritisiert und um nicht-hedonistische Motive ergänzt (Vorderer \& Reinecke, 2015).

Solche ergänzten Vorschläge sind allerdings nicht allesamt gleichermassen fruchtbar, um Unterhaltung zu definieren. Reinecke, Klatt und Krämer (2011) legen beispielsweise dar, dass nicht nur hedonistische Motive, sondern auch psychologische Motive zum Konsum von Unterhaltung führen. Tamborini und Kollegen (Tamborini, Bowman, Eden, Grizzard \& Organ, 2010; Tamborini et al., 2011) verstehen Unterhaltung als Erfüllung innerer Bedürfnisse und zeigen dabei, dass neben intrinsischen, hedonistischen Motiven auch Kompetenz, Autonomie und soziale Eingebundenheit als intrinsische, nicht-hedonistische Motive zu «Enjoyment» führen. Beide Autorenteams messen Enjoyment jedoch anhand traditioneller, affektiver Items wie «macht Spass» oder «ist unterhaltend» (Reinecke et al., 2011, S. 204; Tamborini et al., 2011, S. 1030). Die Diskussion dreht sich entsprechend um die Antezedenzien und Konsequenzen von Unterhaltung, ohne aber den Kern, die Unterhaltung an sich, zu tangieren. Dass Motive wie Erholung, Habitus oder Eskapismus zu Unterhaltung führen, haben schon diverse frühere Arbeiten gezeigt (Bosshart \& Macconi, 1998).

Oliver und Raney (2011) ergänzen die hedonistische Vergnügungsperspektive um eine «eudämonistische» Perspektive der «Sinnhaftigkeit». Ihre Arbeit unterscheidet sich von den eben zitierten dadurch, dass Unterhaltung zweidimensional operationalisiert wird und nicht «nur» durch mehrdimensionale Motive zu erklären versucht wird. Sinnhaftigkeit von Unterhaltung besteht darin, die persönliche Entwicklung zu unterstützen sowie Wahrheit und Erkenntnisse über den Lebensinhalt und Lebenszweck zu gewinnen (u. a. S. 985). Die Autoren entwickelten in 
einem mehrstufigen Verfahren ein Messinstrument für die beiden Unterhaltungsdimensionen (Oliver \& Raney, 2011, S. 992). In einem zweiten Schritt zogen sie die Dimensionen (im Sinne von gesuchten Gratifikationen) heran, um den Konsum unterschiedlicher Unterhaltungsangebote zu erklären. Es zeigte sich, dass eudämonistische Motive stärker mit «seriöser» Unterhaltung wie Non-Fiction, Dramen oder Science-Fiction einhergingen. Der Konsum von Actionfilmen oder Komödien hingegen beruhte stärker auf hedonistischen Motiven. Im Allgemeinen waren aber hedonistische Motive stärker ausgeprägt als eudämonistische Motive (Oliver \& Raney, 2011, S. 991-992). Oliver und Bartsch (2010) stellten in ähnlicher Hinsicht der hedonistischen Unterhaltungsdimension eine Dimension der «Appreciation» zur Seite. Auf Basis dieser Studien versteht A. Bartsch (2017, S. 174) «entertainment $[\ldots]$ as a form of media use that is intrinsically rewarding for the user, which means that it is enjoyed or appreciated for the sake of the media experience itself». Mit Appreciation ist gemeint, dass Unterhaltung nicht nur spassig und lustig, sondern auch bedeutsam, bewegend oder anregend sein kann (Oliver \& Bartsch, 2010).

Reinecke, Vorderer und Knop (2014, S. 4) ergänzen diese «ZweifaktorenModelle» (S. 4) zu Unterhaltung um eine extrinsische Perspektive, wonach der Konsum von Unterhaltung auch das Resultat sozialen Drucks sein kann. Das trifft insbesondere auf «Unterhaltung 2.0»zu. Diese Form der Unterhaltung ergibt sich durch die Interaktion auf sozialen Netzwerken, beispielsweise durch Partizipation oder Produktion. Unterhaltung 1.0 umschreibt im Gegensatz dazu den klassischen Konsum von Inhalten - ob online oder in traditionellen Medien (Trepte \& Reinecke, 2010). Auch hier wird aus der Konzeptualisierung ihres Modells ersichtlich, dass die Autoren vorrangig von den Motiven sprechen, die zur Hinwendung von Unterhaltung führen (Reinecke et al., 2014). Das zeigt sich nicht zuletzt auch bei der Operationalisierung des Begriffs Enjoyment, die sie von Reinecke et al. (2011) übernehmen.

Im Rahmen dieser Arbeit wird Unterhaltung in Anlehnung an Bosshart und Macconi (1998) oder Früh (2003) als etwas verstanden, dessen Erleben dem Betrachter Freude bereitet, kontrollierbar ist und Spannung abbaut. Die Unterhaltung ergibt sich durch eine Botschaft (unterhaltender Brand Content), die - analog zu den Überlegungen betreffend Information - Unterhaltungspotenzial hat. Die «eudämonistische» Perspektive wird ausgeblendet, weil sich Marken primär hedonistischer Unterhaltung bedienen (Vries, Gensler \& Leeflang, 2012) und weil leichte Unterhaltungskost zu einem Grossteil mit hedonistischen Motiven und Mood Management in Verbindung gebracht werden kann (A. Bartsch, 2017, S. 183). Diese Einschränkung ist auch im Hinblick auf das Fazit von Hallahan 
(2018, S. 3) vertretbar, wonach die meisten Menschen Medieninhalte aus Gründen wie Ablenkung, Eskapismus oder Vergnügen konsumieren.

\subsection{Brand Content: Arbeitsdefinition}

Nach der eingehenden Betrachtung der Userperspektive über den U\&G-Ansatz, der getroffenen Auswahl zugunsten der beiden Gratifikationen Information und Unterhaltung und deren Detailbesprechung in den vorangegangenen Kapiteln kann das in Abschnitt 2.5 entwickelte Verständnis von Brand Content nun komplettiert werden:

Als Brand Content sind unternehmensgenerierte Botschaften der Unternehmenskommunikation zu verstehen, die gleichberechtigt eine Unternehmens- und eine Userperspektive umfassen und primär über digitale Kanäle vermittelt werden. Unter der Unternehmensperspektive werden die klassischen, informativen und emotionalen Kommunikationsstrategien verstanden, die dem Aufbau eines funktionalen bzw. emotionalen Markenimages dienen. Unter der Userperspektive wird ein der Botschaft inhärenter Mehrwert für die User verstanden. Dieser Mehrwert wird in der Tradition des U\&G-Ansatzes als Gratifikation bezeichnet. Wesentliche Gratifikationen, die mit dem Konsum von Inhalten einhergehen, sind Information und Unterhaltung. Abbildung 3.3 bildet die Herleitung und Arbeitsdefinition des informativen und unterhaltenden Brand Contents ab.

Wichtig bleibt zu erwähnen, dass weder informative und emotionale Botschaften noch Information und Unterhaltung (jederzeit) trennscharf voneinander abzugrenzen sind. Oftmals werden diese Bestandteile vermischt und kombiniert (z. B. in der Form von Infotainment) bzw. deren Wahrnehmung unterscheidet sich von Individuum zu Individuum (Bosshart, 2007; Koeppler, 2000, S. 350; Kroeber-Riel \& Gröppel-Klein, 2013, S. 613). 

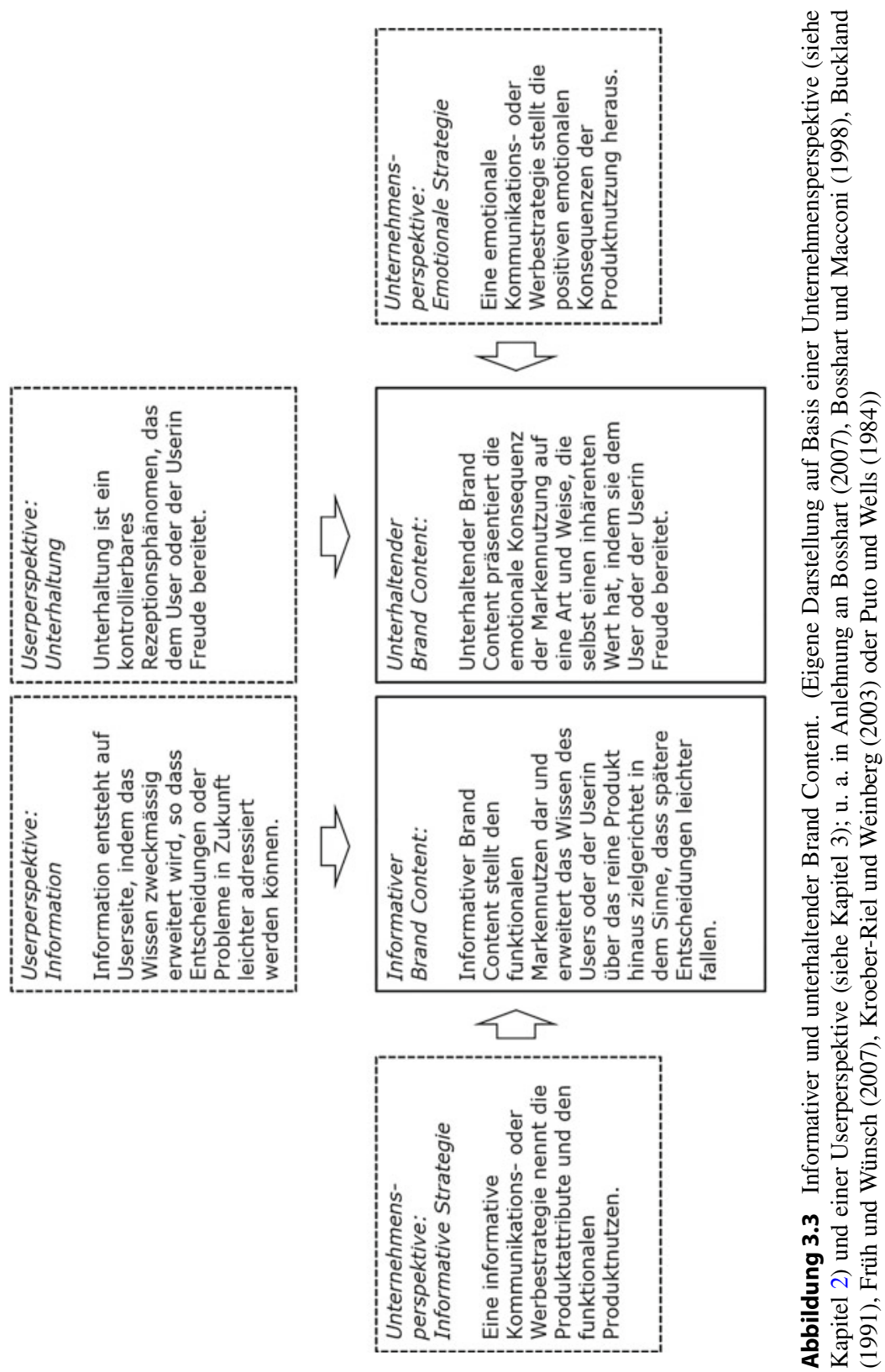
Open Access Dieses Kapitel wird unter der Creative Commons Namensnennung 4.0 International Lizenz (http://creativecommons.org/licenses/by/4.0/deed.de) veröffentlicht, welche die Nutzung, Vervielfältigung, Bearbeitung, Verbreitung und Wiedergabe in jeglichem Medium und Format erlaubt, sofern Sie den/die ursprünglichen Autor(en) und die Quelle ordnungsgemäß nennen, einen Link zur Creative Commons Lizenz beifügen und angeben, ob Änderungen vorgenommen wurden.

Die in diesem Kapitel enthaltenen Bilder und sonstiges Drittmaterial unterliegen ebenfalls der genannten Creative Commons Lizenz, sofern sich aus der Abbildungslegende nichts anderes ergibt. Sofern das betreffende Material nicht unter der genannten Creative Commons Lizenz steht und die betreffende Handlung nicht nach gesetzlichen Vorschriften erlaubt ist, ist für die oben aufgeführten Weiterverwendungen des Materials die Einwilligung des jeweiligen Rechteinhabers einzuholen.

(c) (9) 